\title{
L'ajout d'erreurs : regard sur un épiphénomène du déficit du savoir grammatical en français au postsecondaire en milieu francophone minoritaire
}

\author{
Marie Bernier \\ Université Laurentienne
}

\begin{abstract}
Résumé
Cette étude rend compte des manifestations du déficit du savoir grammatical en français au postsecondaire en examinant les erreurs ajoutées par de nouveaux étudiants universitaires de milieu francophone minoritaire en situation expérimentale de repérage/correction d'erreurs ciblées, avec réflexion à voix haute. Nos questions de recherche concernent la nature des erreurs ajoutés et les raisonnements métalinguistiques qui justifient celles-ci. Les résultats montrent des ajouts d'erreurs de tout type et que les raisonnements des sujets les mènent souvent à douter d'une orthographe correcte. Ils confirment encore les difficultés particulières de l'apprentissage du français normatif en milieu minoritaire et suggèrent que des actions sont à entreprendre à la fois en amont, dans l'enseignement de la langue au secondaire, et en aval, dans la mise en œuvre de modes d'acculturation à l'écrit savant au niveau universitaire.
\end{abstract}

\section{Mots clés}

pédagogie universitaire; savoir métalinguistique; français en milieu minoritaire; savoir grammatical au postsecondaire; français écrit à l'université

\section{Abstract}

This study reports on the phenomenon of adding errors when revising or correcting text, by university freshmen originating from minority French-speaking environments. The study used an experimental situation whereby error corrections were targeted while recording verbally expressed self-reflections. Our research interest focused on the nature of errors added and the underlying reasoning justifying them. Results showed a wide variety of error types and that the reasoning used by subjects led them often to doubt a correct spelling. Findings confirm some of the difficulties encountered in the course of French teaching in minority environments and underline the need for remedial action in high school teaching as well as in teaching undergraduate scientific writing. 


\section{Introduction}

La question de la compétence langagière des étudiants abordant des études postsecondaires semble avoir un potentiel d'actualité infiniment renouvelable, à en croire les nombreuses études qui lui ont été et lui sont encore d'une façon ou d'une autre consacrées (par exemple, Beaudry, 2008; Grégoire, 2011; Lefrançois, 2003; Monballin, 2011; Piron, 2008). Que l'on parle des difficultés d'ordre grammatical et syntaxique, ou encore du développement des habiletés rédactionnelles et pragmatiques, il semble que, malgré les efforts consentis dans le renouvellement de l'enseignement de la langue aux ordres préuniversitaires et les mesures d'aide, dont il faut dire qu'elles donnent certains résultats, comme le soulignent Lefrançois, Laurier, Lazure et Claing (2005), les étudiants des collèges et universités éprouvent de réels problèmes en français écrit et plus particulièrement en regard de l'écrit de type universitaire.

\section{Problématique}

Roy et Lafontaine (1992) ont mis en lumière, il y a vingt ans, lors d'un travail d'analyse sur la maitrise du français aux ordres postsecondaires, un épiphénomène de la méconnaissance de la langue écrite : l'ajout d'erreurs lors du processus de révision/correction. Ce phénomène, généralisé, n'épargnerait selon eux aucun aspect du français écrit. L'ajout d'erreurs, disent les auteurs, « c'est ce qui permet de saisir les diverses facettes qui constituent le tableau ou le casse-tête des fausses certitudes accumulées en français au cours des treize années de formation scolaire » ( $p$. 34). Corriger des formes non fautives revient à dire ne pas connaitre suffisamment les règles gouvernant l'orthographe lexicale et grammaticale pour faire la différence entre forme fautive et non fautive. Cela met en évidence des difficultés qui passent souvent inaperçues, l'attention étant généralement portée sur la (re)connaissance et la mémoire des règles de la grammaire. Cela pose ainsi une fois de plus la question de la compétence en français écrit, particulièrement aux ordres postsecondaires où l'on considère de façon générale que l'apprentissage de base des règles de la langue écrite, tout au moins au plan de l'orthographe grammaticale, devrait être accompli et qu'il faut commencer cet autre apprentissage, l'appropriation d'un certain discours scientifique (Beaudry, 2008; Lebel, 2011; Lefrançois, 2003; Monballin, 2011; Monballin et Legros, 2001; Piron, 2008). Cette problématique est d'autant plus alarmante en milieu francophone minoritaire où le contexte de contact des langues, le constant « tiraillement » vécu entre français vernaculaire et français standard (Laflamme et Reguigui, 2003) et l'insécurité linguistique qui en résulte ont une incidence certaine sur l'appropriation du savoir grammatical. 


\section{Sur la compétence langagière}

Nous avons discuté, dans une précédente recherche (Bernier, 2012) où nous nous sommes intéressée aux réflexions métagrammaticales que les étudiants produisent en situation de révision/correction de texte, de la double problématique de la compétence langagière des nouveaux étudiants universitaires francophones en milieu minoritaire. Nous y avons exposé les principales recherches qui, depuis une vingtaine d'années, ont fait état de la problématique, multidimensionnelle, de la compétence langagière lacunaire des étudiants des ordres postsecondaires (Boudreau, 1995; Lafontaine et Legros, 1995; Lépine, 1995; Moffet, 1995; Monballin, van der Brempt et Legros, 1995; Roy et Boudreau, 1995; Roy, 1992, 1995; Roy et Lafontaine, 1992; Roy et Legros, 1995; Simard, 1995; Vanderdorpe, 1995; Viau, 1995). Si les études que nous mentionnons ici semblent dater, émergées dans les années 1990 à la suite des résultats de l'étude précitée de Roy et Lafontaine (1992), la question des connaissances et des habiletés en français écrit des nouveaux étudiants aux ordres postsecondaires demeure entière, cela malgré les diverses mesures de remédiation mises en place par les collèges et universités, lesquelles visent souvent, en dépit des besoins pressants d'acculturation aux écrits technoscientifiques, l'orthographe grammaticale (Beaudry, 2007, 2008; Grégoire, 2011; Lefrançois, Laurier, Lazure, Claing, 2008; Lefrançois, 2003; Monballin et Legros, 2001; Piron, 2008). Dans cette étude, nous avons aussi exposé les difficultés plus importantes que rencontrent les francophones vivant en milieu minoritaire au regard de l'apprentissage du français, oral ou écrit, puisque l'objet enseigné - le français comme matière scolaire a parfois peu de rapport avec la langue vernaculaire. Aussi n'en reprendrons-nous ici que les grandes lignes.

Il convient tout d'abord de distinguer compétence langagière et compétence linguistique. Selon Laflamme et Reguigui (2003), la compétence linguistique ne représente que l'une des composantes de la compétence langagière, notion multidimensionnelle impliquant les dimensions cognitive, communicationnelle et linguistique. C'est cette dernière dimension, qui renvoie aux « composantes formelles de la langue [...] le lexique, la morphologie, la syntaxe, la phonétique et la phonologie ainsi que la sémantique » (p. 11), qui nous intéresse ici. Nous souscrivons aussi au sens qu'en donne Lépine (1995), pour qui la compétence linguistique résulte de la connaissance suffisamment intégrée des règles et conventions orthographiques régissant le système linguistique afin que leur application soit devenue quasi automatique ou encore qu'elle procède d'une réflexion métalinguistique en adéquation avec le contexte.

Or, ce degré de compétence linguistique se trouve diversement atteint par les étudiants du postsecondaire, les études sur les mesures de remédiation précitées en faisant foi. En particulier, ces études révèlent des lacunes en orthographe grammaticale, où les règles de base de la grammaire des accords ne sont souvent que partiellement maitrisées, en syntaxe et en ponctuation, où l'organisation phrastique présente souvent d'importantes lacunes, notamment au plan des choix lexicaux et à celui de la gestion de la ponctuation syntaxique, et où l'organisation textuelle reflète un savoir lacunaire dans la gestion des marqueurs textuels. La problématique de la compétence linguistique nécessaire et attendue au postsecondaire demeure donc entière, d'autant plus que le fait d'entrer aux études universitaires signifie s'exposer à une culture langagière nouvelle, à une nouvelle utilisation de la langue, à un impératif d'acculturation à la communication scientifique (Monballin, 2011; Descheper et Thyrion, 2008). En ce sens, cela implique devoir développer ses habiletés rédactionnelles, discursives et pragmatiques. Dans tous les cas, la langue écrite, et même orale, est convoquée, et les efforts doivent être mis ailleurs que sur le code linguistique. 


\section{Sur le milieu minoritaire}

Plusieurs des études susmentionnées émanent de milieu francophone majoritaire où l'on constate que, de manière assez généralisée, la compétence désirée en français écrit demeure problématique. Or, les difficultés rencontrées dans l'appropriation du français écrit par les locuteurs francophones en situation minoritaire se trouvent amplifiées du fait de leur vécu ethnolangagier. Car vivre en milieu minoritaire, comme le disent Landry, Deveau et Allard (2006), signifie vivre en contact avec au moins deux langues, celle de la majorité, dominante et vue comme plus prestigieuse, et celle de la minorité, employée surtout dans la sphère des relations sociales informelles ou de proximité, et ressentie comme moins prestigieuse. De nombreuses études (Bernard, 1998; Boudreau, 2001; Boudreau et Dubois, 2009; Coglan et Thériault, 2002; Cormier, 2004; Dagenais et Moore, 2004; Dallaire, 2004; Dallaire et Denis 2003; Duff, 2007; Duquette, 2006; Gérin-Lajoie, 2004; Gérin-Lajoie et Jacquet, 2008; Lebel, 2011; Mc Andrew et coll., 2008; Pilote et Magnan, 2008) se sont intéressées aux caractéristiques et aux enjeux des milieux minoritaires au Canada, traitant tant des questions démographiques et géographiques que des questions sociolinguistiques, culturelles, scolaires et identitaires des francophones canadiens. L'une des questions centrales concerne l'école de milieu minoritaire, qui doit composer, selon Heller (1999), avec les aspects contradictoires à la fois de son mandat de maintien et de sauvegarde de la langue et de la culture francophones et la réalité anglodominante, partout présente. De façon générale, les études portant sur le vécu ethnolangagier en situation francophone minoritaire s'entendent sur le fait que l'insécurité linguistique, que l'on peut définir à la suite de Gérin-Lajoie et Labrie (1999) comme une « [...] conscience qu'il existe une norme exogène, que l'on associe à une région extérieure, qui serait supérieure par rapport à la variété linguistique en usage dans sa propre région » (p. 87), constitue un facteur important ayant un impact sur la compétence langagière. Une vaste étude sociolinguistique (Laflamme et Reguigui, 2003) qui s'est intéressée, entre autres objectifs, aux facteurs pouvant agir sur la maitrise de la langue maternelle d'un groupe majoritaire en comparaison avec celle d'un groupe minoritaire, montre que l'appartenance à l'un ou l'autre groupe a une incidence sur le nombre et le type d'erreurs commises et que l'influence du groupe dans un rapport majoritaire/minoritaire est réelle. L'étude touchait trois langues - anglais, français et arabe - trois nationalités et trois statuts linguistiques majoritaire, minoritaire et de scolarisation - et a été menée auprès de cinq groupes d'étudiants universitaires lors d'une tâche de rédaction : des Canadiens français et des Canadiens anglais, des Français et des Tunisiens, francisants et arabisants. Les auteurs ont constaté une différence significative dans le nombre d'erreurs commises, tous types confondus, entre les locuteurs de milieu majoritaire et ceux de milieu minoritaire. Par exemple, l'échantillon canadienfrançais (milieu minoritaire) a commis presque deux fois plus d'erreurs que l'échantillon français (milieu majoritaire). C'est, disent les auteurs, au plan des composantes formelles de la langue, en morphologie et en syntaxe, que les erreurs sont les plus percutantes chez les sujets de l'échantillon canadien-français.

En Ontario, le programme de français au secondaire pour les écoles francophones définit des attentes et des contenus spécifiques d'apprentissage à atteindre au terme des études secondaires en communication orale, en lecture et en écriture. Selon le Ministère de l'éducation de l'Ontario (MÉO, 2007), les élèves qui sont issus de ses écoles devraient présenter, au moment d'aborder des études postsecondaires, les connaissances linguistiques et textuelles telles que décrites dans son programme Français. Cours préuniversitaire, 88 quant aux aspects suivants : grammaire de la phrase / syntaxe; grammaire du texte / cohérence; orthographe d'accord et ponctuation; lexique et orthographe 
d'usage. Ces aspects évoquent la terminologie de la grammaire rénovée, dont l'application en classe se fait encore attendre pour plusieurs écoles. En effet, bien que depuis 2007, le MÉO ait recommandé, et même prescrit dans son programme cadre, que l'enseignement de la grammaire se fasse dorénavant selon le paradigme (qu'il nomme approche) de la grammaire rénovée et ait mis à la disposition des enseignants des lignes directrices pour la production et la réalisation de matériels pédagogiques, peu de soutien et d'encadrement ont été offerts si ce n'est une sensibilisation auprès des enseignants et enseignantes des niveaux primaire et secondaire lors d'ateliers d'initiation à l'enseignement de la grammaire nouvelle. II n'est donc pas étonnant de constater que ce souhait du législateur ait eu si peu de répercussions réelles en salles de classe. Car il s'agit bien, comme le mentionnent Lord et Chartrand (2010), d'une véritable rupture épistémologique, où, contrairement à la grammaire traditionnelle, en grammaire rénovée, une démarche active de découverte constitue la base d'une approche pédagogique qui met l'accent sur les régularités de la langue. II s'agit d'une conception de l'enseignement-apprentissage qui prend appui sur le structuralisme et sur le concept de jugement de grammaticalité de la grammaire générative et transformationnelle chomskyenne. Elle interpelle les connaissances implicites des apprenants, de même que la notion d'activité métalinguistique propre à la psycholinguistique (Boivin, 2009). Un tel changement de cap demande temps, efforts et suivi appuyés par une véritable volonté politique de réalisation. À notre connaissance, l'école francophone ontarienne ne semble pas encore arrivée à un stade significatif d'une implantation généralisée de la grammaire rénovée, du moins dans le nord de la province.

Sans vouloir prétendre que l'enseignement du français écrit selon le modèle de la grammaire nouvelle est le remède qui mènera les futurs étudiants à une maitrise satisfaisante de la langue écrite, nous pouvons affirmer que, étant donnée la constance des piètres résultats qu'il est donné de voir dans les cours de mise à niveau à l'entrée à l'université, la méthode traditionnelle ne semble pas avoir donné les résultats attendus. En effet, nos propres résultats de recherche (Bernier, 2012) montrent que le savoir grammatical d'une majorité de nos sujets provenant d'écoles ontariennes qui entreprennent des études universitaires n'est pas suffisant pour générer des raisonnements basés sur les règles fonctionnelles de la langue; que leur savoir grammatical s'apparente souvent à un amalgame de pseudoconnaissances, parfois mal intégrées; qu'il y a de sévères carences dans les connaissances déclaratives et procédurales des règles de la grammaire du français écrit, ce qui se traduit par une surutilisation de procédures de substitution et la résolution plus sémantique que métalinguistique des problèmes. Quant à savoir si l'approche différente de la grammaire nouvelle serait à même d'améliorer ces aspects, seules des études plus approfondies sur la nature même des difficultés rencontrées par les apprenants et sur les facteurs en jeu permettront éventuellement de répondre à une telle interrogation.

\section{Position du problème et objectifs de la recherche}

L'ajout d'erreurs n'est qu'un épiphénomène de l'ensemble de la problématique de l'appropriation de l'écrit dans ses composantes formelles, mais il est digne de mention puisqu'il en est aussi un indicateur, au même titre que le sont les difficultés rencontrées en révision/correction de texte. En ce sens, il témoigne aussi des importantes difficultés qu'ont plusieurs nouveaux étudiants en français écrit à leur entrée à l'université. Son étude permet d'entrer un tant soit peu dans le savoir métalinguistique des sujets par le biais des jugements de grammaticalité qu'ils portent 
comme justifications aux fausses corrections données à des formes non fautives. Cela vient encore enrichir notre propre connaissance de cette problématique multifactorielle.

C'est dans cette optique que nous nous intéressons à la nature et à l'étendue de ce phénomène lors du processus de correction, c'est-à-dire, dans un premier temps, quant à la proportion, la distribution, les types d'ajouts et les plans de la morphosyntaxe touchés et, dans un second temps, aux jugements de grammaticalité émis par les sujets lors de l'expérimentation.

\section{Méthodologie}

\section{Sujets et procédure de collecte des données}

Pour les besoins de cette recherche, seize participants, étudiants francophones provenant majoritairement du nord de l'Ontario et âgés entre 17 et 19 ans, inscrits en première année de baccalauréat en sciences humaines ou sociales, ont participé volontairement à l'étude. Ces étudiants ont été recrutés dans le cadre du cours de mise à niveau en français écrit, cours obligatoire pour tous les étudiants des Facultés des humanités et des sciences sociales. Des dispositions ont été prises pour assurer la confidentialité des données et l'anonymat des sujets, et leur consentement libre et éclairé a été obtenu par le biais d'une lettre expliquant les objectifs généraux de la recherche, les modalités et les conditions de participation à l'étude.

Les séances de travail individuelles, d'une durée d'au plus 55 minutes, ont eu lieu au laboratoire des Arts et des sciences du langage de l'Université Laurentienne. Les séances ont été intégralement enregistrées en vidéographie à l'aide du Digital Voice Recorder, avec mémoire transférable à un ordinateur PC, de Sony. Les enregistrements ont été détruits après la collecte des données afin de respecter l'anonymat.

\section{Tâche expérimentale}

Les données utilisées dans cette étude émanent d'une recherche dont la tâche expérimentale visait à observer la capacité des participants à repérer, identifier et corriger 64 erreurs distribuées dans un corpus de 26 phrases détachées et de 7 courts textes, équilibrés quant au nombre de mots et ne contenant pas de mots sortant de l'ordinaire. La tâche se présentait sous forme de documents Word, en deux colonnes, où, à gauche, apparaissaient les textes et, à droite, un espace libre où les sujets devaient inscrire leurs corrections. Le tableau 1 présente un échantillon de textes inclus dans ce document. Les textes ont été adaptés de ceux proposés par le Centre collégial de développement de matériel didactique (CCDMD). Nous avons recueilli la version écrite des corrections, et la version audio/vidéo pour les réflexions que nous avons retranscrites en verbatims.

L'ensemble de la tâche (voir tableau 2) se présentait en trois activités dont les deux premières visaient les erreurs morphosyntaxiques. Celles-ci comptaient pour $87 \%$ du nombre total d'erreurs et étaient réparties dans 26 phrases détachées (Activité 1) et quatre courtes histoires (Activité 2) de façon aussi équilibrée que possible : $43 \%$ et $40 \%$ des erreurs respectivement dans les deux activités. Ces erreurs touchaient, moitié/moitié, le groupe verbal (GV) et le groupe nominal (GN). La troisième activité ciblait les erreurs de grammaire textuelle. Onze erreurs, correspondant à $17 \%$ du nombre total d'erreurs pour l'ensemble des activités, étaient réparties dans trois courtes histoires. 
Tableau 1

Échantillons de la tâche expérimentale : erreurs ciblées dans le groupe verbal et erreurs mélangées dans de courts textes

\begin{tabular}{|l|l|}
\hline $\begin{array}{l}\text { 1. Dès qu'elle entra dans la pièce et qu'elle vie que Pierre } \\
\text { était là, elle eut soudain un regard empreint d'espoir. }\end{array}$ & \\
\hline $\begin{array}{l}\text { 2. Après une randonnée de quatre jours en montagne, ils } \\
\text { rentrèrent tous, épuisés de fatigue mais heureux d'avoir } \\
\text { réussit leur pari }\end{array}$ & \\
\hline $\begin{array}{l}\text { 3. Le changement que mon amie Lucia veut faire dans sa } \\
\text { vie est lourd de conséquences et entraîneront des } \\
\text { bouleversements majeurs. }\end{array}$ & \\
\hline $\begin{array}{l}\text { 4. Les rongeurs sont revenuent dans notre jardin, mais } \\
\text { nous leur avons tendu un piège : notre chat est aux } \\
\text { aguets... }\end{array}$ & \\
\hline $\begin{array}{l}\text { 5. C'est pendant la nuit que les policiers ont téléphonés } \\
\text { les parents pour qu'ils viennent chercher leurs enfants, } \\
\text { enfuis depuis deux jours. }\end{array}$ & \\
\hline
\end{tabular}

\section{COURT TEXTE $4 \sim$ L'INCENDIE}

a) Les lourds nuages aperçus plus tôt n'étaient pas des nuages de pluie mais bien des nuages de fumée. Un voisin nous avaient fait savoir qu'il avait entendu à la radio qu'un incendie de grande envergure s'était déclaré avenue du Parc.

b) Les flammes avaient déjà détruits plusieurs habitations. $\mathrm{Ce}$ n'était pas loin de chez nous, à peine à quelques rues. Les gens commençaient à fuir, allant qui chez des amis, qui chez des voisins qui les accueillais sans posé de questions.

c) Le vent soufflait dans notre direction et attaquerait bientôt les immeubles voisins. L'odeur du feu était envahissante, s'infiltrant partout. On avait pas bien compris comment cette incendie s'était déclaré dans notre quartier, mais le feu était là, présent, effrayant, ravageur.

d) II s'était laissé porté par le vent, on l'avait laissé passer et on le regardait tout détruire sur son passage. Les pompiers travaillaient ferme. Le père de Marie était pompier et je savais qu'il était parfaitement préparé pour ce travail. 


\section{Tableau 2}

Description de la tâche expérimentale

\begin{tabular}{|c|c|c||c|}
\hline \multicolumn{3}{|c||}{ MORPHOSYNTAXE } & GRAMMAIRE TEXTUELLE \\
\hline \hline \multicolumn{2}{|c||}{ ACTIVITÉ 1 } & ACTIVITÉ 2 & ACTIVITÉ 3 \\
\hline 26 phrases détachées & 4 courtes histoires & 3 courtes histoires \\
\hline 21 mots/phrase & 21 mots/phrase & 41 mots/phrase & 54 mots/phrase \\
\hline \hline $\begin{array}{c}14 \text { E distribuées } \\
\text { dans le GV } \\
(50 \%)\end{array}$ & $\begin{array}{c}14 \text { E distribuées } \\
\text { dans le GN (50\%) }\end{array}$ & $\begin{array}{c}25 \text { E distribuées dans } \\
\text { le GV et le GN }\end{array}$ & 11 E distribuées \\
\cline { 2 - 3 } $\begin{array}{c}43 \% \text { E distribuées dans phrases } \\
\text { détachées }\end{array}$ & $\begin{array}{c}40 \% \text { E distribuées } \\
\text { aléatoirement }\end{array}$ & $\begin{array}{c}\text { Référents; connecteurs logiques; } \\
\text { concordance temporelle }\end{array}$ \\
\hline \hline \multicolumn{3}{c|}{$83 \%$} & $17 \%$ \\
\hline
\end{tabular}

\section{Méthodes d'analyse}

Même si la tâche portait sur le repérage et la correction d'erreurs, notre analyse porte uniquement sur les erreurs ajoutées par les sujets. Ainsi, pour répondre à notre premier objectif portant sur la nature et l'étendue du phénomène, nous avons d'abord calculé le ratio moyen d'ajout d'erreurs sur le nombre total de mots lus par les sujets, soit 1264 mots. La distribution de fréquence et les pourcentages ont ensuite été établis en fonction de six catégories de difficultés : les accords fautifs dans le groupe verbal, les constructions syntaxiques fautives, les faux accords dans le groupe nominal, les corrections fautives de l'orthographe lexicale, les erreurs touchant la morphologie du verbe et les confusions dans les catégories lexicales.

En ce qui concerne l'analyse des jugements de grammaticalité, les catégories nous ont été inspirées par celles de Roy et Lafontaine (1992) et par ce que nous ont révélé les corrections apportées dans le corpus, que nous avons regroupées selon le facteur métalinguistique tel que défini par Gombert (1990). L'analyse des verbatims a révélé deux grandes tendances : les raisonnements avec ou sans métalangage.

\section{Résultats}

Les résultats se présentent en deux parties. Nous examinons d'abord les types d'erreurs ajoutées par les participants pour ensuite nous attarder aux justifications énoncées pour ces ajouts.

\section{Types d'erreurs ajoutées}

Pendant le processus de repérage et de correction des erreurs constituant la tâche expérimentale, l'ensemble des 16 sujets a ajouté 213 erreurs sur des formes non fautives, ce qui signifie qu'il y a introduction d'une erreur tous les 5,9 mots en moyenne pour l'ensemble de la tâche réalisée, c'est-à-dire sur 1264 mots. En d'autres termes, la potentialité d'ajout est de 0,16 erreur par mot. Nous présentons au tableau 2 la distribution des ajouts d'erreurs pour chacune des 6 catégories d'analyse retenues. La lecture de ce tableau montre que l'ajout d'erreurs se fait principalement au plan des accords grammaticaux et de la syntaxe, avec $85,9 \%$ des ajouts au total. Ce pourcentage inclut tant les ajouts d'erreurs d'accords que ceux concernant la construction syntaxique. 
Tableau 3

Nombres et pourcentages d'erreurs ajoutées par catégories

\begin{tabular}{|c|c|c|c|c|c|}
\hline \multicolumn{7}{|c|}{ Accords grammaticaux et syntaxe } & Orthographe \\
\hline $\begin{array}{c}\text { Accords fautifs } \\
\text { dans le GV : } \\
\text { participe passé } \\
\text { CompV }\end{array}$ & $\begin{array}{c}\text { Construction syntaxique } \\
\text { fautive Construction } \\
\text { prépositionnelle dans } \\
\text { GN ou dans GV }\end{array}$ & $\begin{array}{c}\text { Faux accords } \\
\text { dans le GN : } \\
\text { genre/nombre } \\
\text { Dét/Nom/ } \\
\text { Nom/Adj }\end{array}$ & $\begin{array}{c}\text { Morphologie du } \\
\text { verbe } \\
\text { Conjugaison } \\
\text { Concordance } \\
\text { temporelle }\end{array}$ & $\begin{array}{c}\text { Confusion } \\
\text { catégorie } \\
\text { lexicale : } \\
\text { faux } \\
\text { accords } \\
\text { Adj/Adv }\end{array}$ & $\begin{array}{c}\text { Corr. fautives } \\
\text { orthographe } \\
\text { lexicale }\end{array}$ \\
\hline 58 & 53 & 32 & 23 & 17 & 30 \\
\hline $27,2 \%$ & $24,9 \%$ & $15,0 \%$ & $10,8 \%$ & $8,0 \%$ & $14,1 \%$ \\
\hline
\end{tabular}

Par ordre d'importance, en ce qui a trait aux accords grammaticaux et à la syntaxe, c'est dans le groupe verbal que l'on retrouve la plus importante proportion d'erreurs ajoutées. II s'agit d'ajouts relatifs à l'accord des participes passés ou du complément du verbe, qui représentent presque le tiers des ajouts $(27,2 \%)$. La syntaxe est le deuxième lieu où les sujets ont manifesté une tendance évidente à l'ajout d'erreurs, avec le quart de tous les ajouts $(24,9 \%)$ de notre corpus. On parle ici de construction phrastique, et il s'agit le plus souvent de difficultés reliées aux phrases complexes, où, par exemple, le verbe se trouve séparé de son sujet par un groupe complément du type subordonné, ou de groupes syntaxiques déplacés par rapport à leur ordre naturel. Les sujets ne semblent pas savoir reconstruire la phrase selon les groupes syntaxiques, ne serait-ce que de premier niveau. La construction prépositionnelle dans le groupe complément du nom, qui relève souvent du régime verbal mais qui apparait aussi dans le groupe nominal, semble aussi poser problème aux sujets. Viennent ensuite les ajouts d'erreurs dans le groupe nominal. Ceux-ci relèvent principalement d'accords fautifs en genre et en nombre du nom avec le déterminant ou de l'adjectif avec le nom. Ce type d'ajouts représente 15,0\% de l'ensemble des ajouts. Pour leur part, les ajouts relatifs à la morphologie verbale, en conjugaison et en concordance temporelle, totalisent, dans notre corpus, $10,8 \%$ des ajouts. II est à noter ici que cette partie de la tâche était proportionnellement moins importante. Enfin, les ajouts relatifs à des accords fautifs dus à une confusion entre des catégories lexicales, notamment adjectivale et adverbiale, se présentent dans une proportion de $8,0 \%$.

Le 14,1 \% d'ajout d'erreurs restant est relatif à l'orthographe lexicale, ce qui représente une proportion non négligeable des ajouts, surtout si l'on se rappelle qu'il n'y avait pas, dans le corpus de la tâche, de mots sortant de l'ordinaire. II s'agit des géminées, de la finale de mots féminins comme beauté, des titres et fonctions qui constituent des lieux d'ajout d'erreurs de la part des sujets.

Ces résultats montrent que, lors du processus de correction, les sujets manifestent une tendance à l'ajout d'erreurs essentiellement en orthographe grammaticale (morphosyntaxe). Ces ajouts ont eu lieu principalement dans le groupe verbal, puis en syntaxe, en construction phrastique et prépositionnelle. Nous voulons maintenant examiner ces résultats à la lumière des raisonnements métagrammaticaux émis par les sujets pendant la réalisation de la tâche 


\section{Justification des ajouts d'erreurs par les sujets}

L'ajout d'erreurs est le fait, pour un scripteur, de changer volontairement la graphie juste d'un mot qu'il juge fautif à la suite d'un raisonnement. Selon Gombert (1990), rappelons-le, ce raisonnement implique que le sujet utilise un métalangage pour rendre compte de son activité consciente de réflexion sur la langue et son utilisation. Ce métalangage représente les connaissances grammaticales acquises par le sujet, qu'elles soient sûres ou chancelantes. Voyons donc quels sont les ajouts et les raisonnements qui sont à leur origine.

Morphosyntaxe (orthographe grammaticale)

Tableau 4

Types d'erreurs ajoutées dans le groupe verbal et exemples de raisonnement ${ }^{1}$

\begin{tabular}{|c|c|}
\hline Types d'erreurs ajoutées & Raisonnements \\
\hline $\begin{array}{l}\text { 1. Accords verbe/sujet } \\
\text { - conjuguer le verbe en personne, temps et } \\
\text { mode }\end{array}$ & $\begin{array}{l}\text { les pires moments, je les vis } \\
\text { - je vit, donc } \mathrm{V}-\mathrm{l}-\mathrm{T} . . . \mathrm{hmm} \text {... je fais la réécriture mais je suis pas } \\
\text { certaine. Hmmm whatever je vais le remettre (mais a laissé sa } \\
\text { correction) }\end{array}$ \\
\hline - accorder le verbe à son sujet & $\begin{array}{l}\text { on parle de plus en plus de l'importance du recyclage } \\
\text { - parl-E-N-T parce qu'il y a plus qu'une personne }\end{array}$ \\
\hline $\begin{array}{l}\text { - distinguer le sujet et le complément du } \\
\text { verbe }\end{array}$ & $\begin{array}{l}\text { ce qui lui a coûté une forte amende (à Sylvie) } \\
\text { - coûté... hmm... au féminin? Oui, j'vais le mettre au féminin parce que } \\
\text { c'est Sylvie } \\
\text { - a coûT-É-E parce que c'est une interdiction de stationner }\end{array}$ \\
\hline - reconnaître un verbe impersonnel & $\begin{array}{l}\text { il leur fallait un permis } \\
\text {-qu'llS avec un -S parce que c'est les deux entrepreneurs }\end{array}$ \\
\hline - reconnaître la forme infinitive & $\begin{array}{l}\text { les hommes [...] ont commencé à se battre } \\
\text {-les hommes, donc à se battrE- } N-T \text { au pluriel }\end{array}$ \\
\hline - accorder avec un nom non comptable & $\begin{array}{l}\text { de l'information est disponible } \\
\text {-on doit dire SONT disponibles (sans justification) }\end{array}$ \\
\hline $\begin{array}{l}\text { 2. Accords des participes passés } \\
\text { - distinguer la forme participiale de la forme } \\
\text { non participiale }\end{array}$ & $\begin{array}{l}\text { erreurs que j'ai commises } \\
\text {-sans -S parce que c'est la } 1 \text { e personne du singulier, pas de }-S \\
\text { nous n'avons pas prêté attention (deux femmes) } \\
\text {-prêté-É-s parce que c'est deux femmes }\end{array}$ \\
\hline $\begin{array}{l}\text { - reconnaitre les auxiliaires de conjugaison } \\
\text { de formes homophoniques, tels ont/on, } \\
\text { sont/son }\end{array}$ & $\begin{array}{l}\text { la formation qu'ils ont reçu(s) } \\
\text { ils ont... hmmm... j'me trompe toujours avec les }-\mathrm{O}-\mathrm{N}-\mathrm{T} \text { mais je pense } \\
\text { que c'est O-N }\end{array}$ \\
\hline 3. Concordance temporelle & $\begin{array}{l}\text { depuis longtemps, les Hollandais et les Allemands participent } \\
\text { - particip-A-I-E-N-T parce que c'est depuis longtemps, alors à l'imparfait } \\
\text { se sont levés et ont commencé à se battre } \\
\text {-commen-C-E-N-T parce que ça suit l'action }\end{array}$ \\
\hline
\end{tabular}

1 II faut lire ainsi les verbatims : les énoncés sont repris tels qu'ils ont été écrits au fur et à mesure de la tâche. Nous avons ajouté les majuscules pour identifier les parties que les sujets épelaient. Les termes où il y a eu ajout d'erreur sont en italique dans l'énoncé source. Nous avons mis les erreurs ciblées entre parenthèses puisque c'est ce que les sujets voyaient. 
Nos données montrent que le plus difficile pour les sujets demeure ce qui concerne le verbe, qu'il s'agisse des accords (verbes prédicats et participes passés), de la conjugaison ou de la concordance temporelle. L'une des difficultés souvent rencontrées à cet égard concerne l'identification de la forme verbale en soi, ce qui n'aide en rien la capacité d'établir de façon claire les accords à effectuer. De plus, les sujets éprouvent certaines difficultés, une fois le prédicat bien identifié, à établir, dans un premier temps, s'il y aura accord ou non, et dans un second temps, où, comment, avec quel groupe/mot se fera cet accord. II est ainsi apparu que l'accord du prédicat avec le sujet, dès lors qu'il y avait un écran ou que le verbe revêtait la forme composée, recelait des difficultés importantes, comme en témoignent les raisonnements qui ont donné lieu à ces « corrections » dans les exemples présentés au tableau 4. De même, la confusion complément du verbe/sujet représente un réel problème pour de nombreux sujets quand vient le moment d'établir s'il y aura ou non accord, et surtout avec lequel des deux. Souvent, c'est la stratégie de la position qui prévaut : on accordera le prédicat avec le mot qui est placé devant, nonobstant sa fonction syntaxique. Une autre difficulté rencontrée par les sujets a trait à la reconnaissance du verbe à la forme participiale ou, si l'on veut, au participe passé comme tel. Dès lors qu'il y a un écran entre l'auxiliaire et le participe adjectif, c'est comme si les sujets n'avaient pas la capacité de le reconnaitre, ce qui résulte souvent en un accord du participe adjectif comme s'il était le prédicat. Le tableau 4 présente des exemples qui touchent les accords verbe/sujet; l'accord du participe passé; et la concordance temporelle. On le voit, les réflexions ne reposent pas sur des justifications grammaticalement appropriées, bien qu'elles utilisent généralement les mots du métalangage.

Tableau 5

Types d'erreurs d'accords dans le groupe nominal et exemples de raisonnements

\begin{tabular}{|c|c|}
\hline Types d'erreurs ajoutées & Raisonnements \\
\hline $\begin{array}{l}\text { 1. Accords nom/déterminant et } \\
\text { adjectif/nom avec tout, chaque }\end{array}$ & $\begin{array}{l}\text { tous le monde } \\
\text { - tous, c'est TOUTE car c'est toute le monde (par euphonie) } \\
\text { chaque personne voulai(ent) } \\
\text { - personne avec un -S à cause que c'est comme si tu disais «tout le } \\
\text { monde», donc pluriel }\end{array}$ \\
\hline $\begin{array}{l}\text { 2. Accord avec un nom collectif ou non } \\
\text { comptable }\end{array}$ & $\begin{array}{l}\text { des contenants de verre } \\
\text { - verre avec un -S parce que il y en a beaucoup } \\
\text { les ordures de tous ordres } \\
\text { - je crois pas que tous ça soit un -S... je suis pas bonne avec les } \\
\text { tout; c'est pas touS comme «on a touS» c'est touT ordre. Aussi, } \\
\text { ordre m'a l'air un peu bizarre } \\
\text { ceux en plastique } \\
\text { - je pense que «plastique» prend un -S parce que c'est «ceux» en } \\
\text { plastiqueS }\end{array}$ \\
\hline $\begin{array}{l}\text { 3. Accord du complément du nom, par } \\
\text { exemple, un accord « grammatical » de } \\
\text { l'adjectif complément du nom }\end{array}$ & $\begin{array}{l}\text { le président Obama, nouvelle star internationale } \\
\text { - nouvel juste un L pas deux. Nouveau? hmmm. Sais pas. } \\
\text { - Nouvelle ici, ça devrait être au masculin parce que c'est Barak } \\
\text { Obama qui est nouvel } \\
\text { il est resté plein d'amertume } \\
\text { - plein avec un S... je suis pas certaine mais je sais que certains } \\
\text { mots prend toujours des S. Mais je suis pas certaine }\end{array}$ \\
\hline
\end{tabular}


Le problème de l'accord du verbe est fondamental, car il conditionne tous les autres, ou presque. Ce qui ressort ici, c'est, encore une fois, l'indifférenciation à la fois au plan de la catégorie lexicale et au plan de la fonction syntaxique. Pour plusieurs, les réflexions pour en déterminer l'accord sont basées sur la position des mots, quelle que soit par ailleurs leur classe ou leur fonction syntaxique. On voit aussi que, concernant le verbe, une vérité semble incontournable pour les sujets : si c'est un verbe, il faut « l'accorder avec quelque chose ».

Si la gestion du verbe et ses accords représente la majorité des ajouts d'erreurs dans le corpus de notre étude, les accords régis par le nom présentent aussi des difficultés pour les sujets. Les ajouts d'erreurs dans les accords nom/déterminant et adjectif/nom montrent que les sujets ont de la difficulté à identifier les catégories et s'appuient le plus souvent sur des raisonnements sémantiques pour justifier leur correction. Les erreurs typiques avec tout, chaque, l'accord avec un nom collectif ou non comptable, l'accord ou non du complément du nom, par exemple, un accord « grammatical » de l'adjectif complément du nom en compétition avec un accord plus sémantique, sont les lieux où les sujets ont le plus souvent trébuché. Ce sont des réflexions le plus souvent basées sur la sémantique, sans égard à la grammaticalité. Le tableau 5 en présente quelques exemples.

Tableau 6

Types d'erreurs d'orthographe lexicale et exemples de raisonnements

\begin{tabular}{|l|l|}
\hline \multicolumn{1}{|c|}{ Types d'erreurs ajoutées } & \multicolumn{1}{c|}{ Raisonnements } \\
\hline 1. Géminées & $\begin{array}{l}\text { ma facilité d'expression } \\
\text { - facilité avec un E je pense } \\
\text { qu'un incendie de grande envergure } \\
\text { - UNE incendie, il y a un } E \text { à la fin }\end{array}$ \\
\hline $\begin{array}{l}\text { 2 Morphologie des noms, tant au féminin } \\
\text { qu'au masculin }\end{array}$ & $\begin{array}{l}\text { mon sens de la répartie } \\
\text { - c'est UN sens, donc pas de } E \text { à répartie }\end{array}$ \\
\hline 3. Mots homophoniques de classes différentes & $\begin{array}{l}\text { après avoir réglé le différend } \\
\text {-je crois que différend, ici, c'est avec un T pas un } D\end{array}$ \\
\hline 4. Féminisation de titres et fonctions & $\begin{array}{l}\text { ces deux entrepreneures } \\
\text { - entrepreneures pas de } E \text {, c'est UN entrepreneur }\end{array}$ \\
\hline
\end{tabular}

Orthographe lexicale (d'usage)

En ce qui a trait aux ajouts d'erreurs en orthographe lexicale, elles concernent bien sûr les géminées, une difficulté répandue, mais aussi et surtout la morphologie des noms au féminin, du type beauté/(beautée) ou banlieue/(banlieuse), la féminisation de titres et fonctions, du type entrepreneur/entrepreneure, ou encore de mots homophoniques mais de classes différentes, tels différend/différent. De même, l'orthographe de l'adverbe construit sur l'adjectif présente aussi des problèmes, par exemple violemment/violament. Le tableau 6 en fournit des exemples. L'orthographe lexicale française comporte des difficultés, et même les scripteurs experts consultent leurs ouvrages de référence à un moment ou à un autre. Sauf pour la féminisation du terme entrepreneure, les réflexions émises nous amènent à penser que, même lorsqu'il ne s'agit pas de morphosyntaxe, on cherche une façon d'établir un accord. Encore une fois, il semble que les sujets de notre étude ne savent pas différencier l'orthographe lexicale de 
l'orthographe grammaticale et que, en tout état de cause, il s'agit de « trouver » un accord, une justification, comme s'il s'agissait d'une sorte de chasse à la faute.

II ressort de ces quelques exemples que les raisonnements à l'origine des ajouts d'erreurs s'apparentent à ceux qui ont prévalu lors de l'exécution de la tâche de révision/correction (Bernier, 2012) : on assiste à une sorte de floraison de raisonnements issus des fausses généralisations, des connaissances lacunaires, embrouillées. De la difficulté à évaluer correctement des formes ou des constructions fautives à l'ajout d'erreurs sur des formes non fautives, les raisonnements sont les mêmes: majoritairement faits de métalangage, mais forcément toujours inappropriés lorsqu'il s'agit d'ajout d'erreurs : raisonnements non grammaticaux, logico-sémantiques, basés sur la reconnaissance visuelle, du type il me semble que ça marche pas, ou encore jugements par analogie sémantique ou euphonique, du type je trouve que ça sonne mal, rappel ou généralisation de règles, raisons invoquées n'ayant souvent rien à voir avec la forme non fautive. Tout cela témoigne d'un enchevêtrement de savoirs qui tiennent à la fois de l'acquis, parcellaire, et de savoirs de locuteurs natifs. Une grammaire dont l'enseignement ne repose pas sur une compréhension du système de la langue et ses régularités a de fortes chances de résulter en un amalgame de constructions erronées qui vont s'ancrer dans le savoir parce que données, reçues et perçues comme vraies.

\section{Discussion}

L'ajout d'erreurs ne constitue en fait qu'un épiphénomène, aussi dramatique dans sa manifestation que peut l'être l'incapacité à détecter et à corriger des formes fautives. II rend compte d'un problème plus vaste de noncompétence linguistique dont il témoigne indirectement : l'ajout d'erreurs comme reflet de « ce qui ne va pas » dans la compréhension de la grammaire et, plus globalement, dans le système linguistique tout entier. La focalisation sur cet aspect de la compétence linguistique pose ou re-pose la question de la pédagogie de la grammaire, tant aux ordres préuniversitaires qu'universitaire.

Concernant nos résultats, deux remarques s'imposent. Tout d'abord l'exercice en lui-même, où les sujets avaient à " trouver » les erreurs disséminées dans le corpus, peut avoir eu un effet que l'on pourrait qualifier d'« entrainement », créant ainsi une surenchère dans le repérage. Comme si, dans un tel contexte, l'élève ou l'étudiant mettait tous ses circuits en route et déployait tous ses savoirs dans le but de débusquer « la faute ». Quoi qu'on en dise, la pédagogie du français est encore souvent soumise à cette représentation idéale, pour ne pas dire idyllique, du français, véhiculée par la norme. En pédagogie scolaire, une telle représentation de la langue conduit traditionnellement - ou encore trop souvent et particulièrement en milieu minoritaire - à l'utilisation régulière d'exercices de grammaire, souvent répétitifs et ennuyeux, mais surtout vidés de toute substance communicationnelle et pragmatique, tant au niveau de l'apprentissage de nouvelles règles que lors des inévitables activités de révision/correction, comme si là résidait la solution aux maux de l'apprentissage de la langue. Les études récentes de Lord et Chartrand (2010) et de Chartrand (2011) en témoignent, où il apparait entre autres que la dictée figure encore parmi les exercices récurrents en grammaire, réputée être l'exercice par excellence à la fois de l'apprentissage des règles et de l'évaluation de cet apprentissage. II en résulte que, de l'enseignement de règles à l'utilisation de la langue à des fins communicationnelles, le pont ne se fait pas toujours et que la métalangue finit par n'être qu'un lexique dont les termes prennent des connotations douteuses ou sont vidés de leur sens pour les scripteurs. 
Puis, il faut considérer le contexte sociolinguistique dans lequel l'enquête a eu lieu. En milieu minoritaire, une pédagogie « de la faute » ou de la grammaire traditionnelle intensive peut faire des ravages. Bien que l'ajout d'erreurs ne soit pas un phénomène exclusif au contexte minoritaire, il faut mentionner que ce contexte, comme l'ont souligné Laflamme et Reguigui (2003), a des effets sur l'appropriation de la langue première, et donc sur la compétence langagière. Le contexte diglossique, pour ne pas dire triglossique, si l'on considère l'écart existant entre le français standard enseigné (et valorisé) et le vernaculaire, plus l'environnement anglodominant, ont un impact certain sur cette appropriation. L'enseignement du français supposé standard se trouve donc en compétition directe avec le vernaculaire et la langue dominante, partout présente et vécue comme plus prestigieuse. Le phénomène de l'ajout d'erreurs constitue ainsi un indice supplémentaire à prendre en compte dans l'évaluation de la compétence linguistique et, plus globalement, de la compétence langagière. En pédagogie de la langue, tenir compte de l'ajout d'erreurs au même titre que des difficultés de reconnaissance et d'identification d'erreurs signifierait amorcer un changement de vision de l'enseignement de la langue écrite en tablant plutôt sur l'acquis que sur les manques et surtout, en enseignant comment fonctionne le système linguistique du français plutôt que l'enseignement (et parfois le surenseignement) décontextualisé de règles de grammaire. En ce sens, le paradigme de la grammaire contemporaine issue de la grammaire générative constitue une avenue intéressante par son approche systémique et plus scientifique des phénomènes linguistiques.

Les mesures de remédiation offertes par les collèges et les universités ont généralement pour mandat et objectif ultime de combler les manques, selon la croyance que la maitrise des aspects formels de la langue entrainera la maitrise des discours convoqués à ce niveau d'études, en oubliant que ces savoirs ne peuvent s'acquérir que par une formation spécifique et continue. Ces mesures en français écrit, cours de mise à niveau inclus, relèvent souvent d'une conception instrumentale de la langue et sont issues, selon le terme de Delcambre et Lahanier-Reuter (2010), d'un discours « défectologique ». Or, selon Laflamme et Reguigui, « [...] en première année d'université, l'erreur linguistique est courante et l'on n'a pas affaire à un phénomène localisé (2003, p. 206) ». Pourtant, comme l'expriment encore Delcambre et Lahanier-Reuter (2010), les difficultés d'écriture des étudiants universitaires sont perçues comme un déficit de la maitrise du code alors qu'il y a un besoin d'appropriation d'un tout nouveau discours, centré sur la connaissance et la diffusion de la connaissance. II s'agirait donc, aux études postsecondaires, de transformer le rapport à l'écrit et la perception qu'en ont les principaux acteurs, étudiants comme professeurs. De nouvelles perspectives se sont fait jour dans le champ de la littéracie universitaire qui aborde l'acculturation à l'écrit universitaire comme un processus nécessitant un apprentissage propre à chaque domaine, et non plus comme un savoir déjà acquis.

\section{Conclusion}

Notre enquête sur l'ajout d'erreurs est une autre illustration des difficultés encore éprouvées par des scripteurs francophones au terme de plus de dix ans d'enseignement-apprentissage de la langue. Elle met aussi en lumière leurs réflexions métalinguistiques qui, elles, ont beaucoup à nous apprendre sur la façon d'enseigner et sur la façon dont est reçu cet enseignement, puisqu'elles donnent un certain accès à la pensée métalinguistique résultant de cet enseignement-apprentissage. Notre étude, bien que non généralisable en raison de la taille de l'échantillon et du 
contexte sociolinguistique, démontre malheureusement une déficience certaine dans les connaissances de base de la langue nécessaires à la compétence requise pour accéder à un autre niveau, celui de l'appropriation de discours universitaires disciplinaires, qui exigent une utilisation différente et différenciée de la langue.

Tout en souscrivant à cette vision, nous croyons aussi qu'il convient d'aller voir en amont et d'observer ce qui se fait réellement en classe de français aux niveaux pré-universitaires et, particulièrement en contexte minoritaire, de poser certaines questions, telles: comment est abordé l'enseignement de la langue? Quelle est la philosophie derrière les choix didactiques? Comment les enseignants perçoivent-ils leur discipline? Comment les futurs enseignants de français sont-ils formés dans un tel contexte? Et, finalement, comment les vœux du ministère de l'Éducation, en ce qui a trait à l'enseignement de la grammaire contemporaine ou rénovée, dont l'approche se veut plus inductive, sont reçus, suivis, et mis en actes? En aval, au niveau post-secondaire, il est à souhaiter que les nouvelles perspectives en pédagogie universitaire en rapport avec les littéracies universitaires, les modes d'aide en français écrit se muent en meilleure utilisation des ressources disponibles, et que l'enseignement de l'écrit dépasse la mise à niveau et soit un point de convergence entre remédiation et apprentissage de l'écrit savant.

\section{Références}

Beaudry, N. (2007). Le RUSAF s'affiche. Correspondance, 13(4), septembre 2007, 1-7.

Beaudry, N. (2008). Définir les attentes en termes de maîtrise et de qualité du français oral et écrit à l'université : des compétences communicationnelles et langagières. Correspondance, 13(4), 1-10.

Bernard, R. (1998). Le Canada français : Entre mythe et utopie. Ottawa : Le Nordir.

Bernier, M. (2012). Justifier faussement la correction ou la non-correction d'erreurs morphosyntaxiques : réflexions métagrammaticales d'étudiants francophones universitaires en milieu minoritaire, Francophonies d'Amérique, 33, 45-78.

Boivin, M.-C. (2009). Manipulations syntaxiques et jugements de grammaticalité dans le travail en classe d'élèves du secondaire. Dans J. Dolz et C. Simard (Dir.), Pratiques d'enseignement grammatical. Points de vue de l'enseignant et de l'élève (p. 179208). Québec : Presses de l'Université Laval.

Boudreau, A. (2001). Langue(s), discours et identité. Francophonies d'Amérique, 12, 93-104.

Boudreau, A. et Dubois, L. (2009). L'insécurité linguistique comme entrave à l'apprentissage du français. Dans M. Ali-Khodja et A. Boudreau (Dir.) Lectures de l'Acadie: une anthologie de textes en sciences humaines et sociales, 1960-1994 (p. 469-485). Montréal : Fides.

Boudreau, G. (1995). Processus cognitifs des étudiants du postsecondaire en production de textes. Revue des sciences de l'éducation, 21(1), 75-93.

Centre Collégial de Développement de Matériel Didactique (CCDMD). Amélioration du français. http://www.ccdmd.qc.ca/fr/

Chartrand, S.-G. (2011). Recension de Des objets enseignés en classe de français par B. Schneuwly et Joaquim Dolz. Presses Universitaires de Rouen, Revue suisse des sciences de l'éducation.

Coglan, V. et Thériault, J.-Y. (2002). L'apprentissage du français en milieu minoritaire, une revue documentaire. Ottawa : CIRCEM.

Cormier, M. (2004). Finalités justes ou attentes démesurées? Le débat autour de l'école en milieu minoritaire. Francophonies d'Amérique, 17, 55-63.

Dagenais, D. et Moore, D. (2004). Représentations ordinaires du plurilinguisme, transmission des langues et apprentissage chez des enfants, en France et au Canada. Langages, 154, 34-46.

Dallaire, C. (2004). Fiers de qui on est... nous sommes francophones ! L'identité des jeunes aux jeux franco-ontariens. Francophonies d'Amérique, 18, 127-147.

Dallaire, C. et Denis, C. (2003). Pouvoir social et modulations de l'hybridité au Canada : les jeunes aux Jeux de l'Acadie, aux Jeux franco-ontariens et aux Jeux francophones de l'Alberta. Études canadiennes, 29(55), 7-23.

Delcambre, I. et Lahanier-Reuter, D. (2010). Les littéracies universitaires : Influence des disciplines et du niveau d'étude dans les pratiques de l'écrit. Dans C. Blaser et M. Ch. Pollet (Dir.) L'appropriation des discours universitaires. Diptyque, 18, 11-42. Namur : Presses Universitaires de Namur.

Deschepper, C. et Thyrion, F. (2008). L'entrée dans le supérieur et l'accès aux discours universitaires : opérationnaliser la notion de rapport à l'écrit dans un projet de formation. Dans $\mathrm{S}$. Chartrand et $\mathrm{C}$. Blaser (Dir.). Le rapport à l'écrit : un outil pour enseigner de l'école à l'université. Dyptique, 12, 61-75. Namur : Presses Universitaires de Namur. 
Duff, P. (2007). Multilingualism in Canadian schools : Myths, realities, and possibilities. Canadian Journal of Applied Linguistics, 10(2), 149-163.

Duquette, G. (2006) Le bilinguisme des élèves inscrits dans les écoles secondaires de langue française de l'Ontario : perceptions, valeurs et comportement langagier. Revue des sciences de l'éducation, 32(3), 665-689.

Gérin-Lajoie, D. (2004). La problématique identitaire et l'école de langue française en Ontario. Francophonies d'Amérique, 18, 171179.

Gérin-Lajoie, D. et Jacquet, M. (2008). Regards croisés sur l'inclusion des minorités en contexte scolaire francophone minoritaire au Canada. Éducation et francophonie, 36(1), 25-43.

Gérin-Lajoie, D. et Labrie, N. (1999). Les résultats aux tests de lecture et d'écriture en 1993-1994: une interprétation sociolinguistique. Dans N. Labrie et G. Forlot (Dir.). L'enjeu de la langue en Ontario français (p. 79-108). Sudbury: Prise de parole.

Gombert, J.-É. (1990). Le développement métalinguistique. Paris : Presses universitaires de France.

Grégoire, C. (2011). Réécriture et compétences langagières. Correspondance, 11(3), 1-2.

Heller, M. (1999). Quel(s) français et pour qui? Discours et pratiques identitaires en milieu scolaire franco-ontarien. Dans N. Labrie et G. Forlot (Dir.). L'enjeu de la langue en Ontario français (p. 129-165). Sudbury : Prise de parole.

Laflamme, S. et Reguigui, A. (2003). Homogénéité et distinction. Sudbury : Prise de parole.

Lafontaine, L. et Legros, C. (1995). Profils linguistiques, cognitifs et motivationnels d'étudiants du postsecondaire faibles en français écrit. Revue des sciences de l'éducation, 21(1), 121-144.

Landry, R., Deveau, K. et Allard, R. (2006). Langue publique et langue privée en milieu ethnolinguistique minoritaire : les relations avec le développement psycholangagier. Francophonies d'Amérique, 22, 167-184.

Lebel, M.-É. (2011). L'enseignement du français à l'Université de Moncton : une formation axée sur les genres de l'écrit et de l'oral. Correspondance, 16(2), 1-6.

Lefrançois P. (2003). Pour le rehaussement de la formation linguistique à l'université. L'autre forum, 8(1), 9-13.

Lefrançois, P., Laurier, M.-D., Lazure, R. et Claing, R. (2008). Évaluation de l'efficacité des mesures visant à l'amélioration du français écrit du primaire à l'université. Montréal : Office québécois de la langue française.

Lefrançois, P., Laurier, M.-D., Lazure, R. et Claing, R. (2005). Les mesures d'aide en français et leurs effets : y a-t-il une recette gagnante ? Correspondance, 11(2), 1-8.

Lépine, F. (1995). Bilan des tests de français à l'admission aux universités québécoises (1987-1994). Revue des sciences de l'éducation, 21(1), 17-35.

Lord, M.-A. et Chartrand, S.-G. (2010). À la recherche de la grammaire rénovée : enquête sur l'enseignement grammatical au secondaire québécois. La Lettre de l'AIRDF, 45(16), 25-33.

McAndrew, M., Garnett, B., Ledent, J., Ungerleider, C., Adumati-Trache, M. et Ait-Said, R. (2008). La réussite scolaire des élèves issus de l'immigration : une question de classe sociale, de langue ou de culture? Éducation et francophonie, 36(1), 177-196.

Ministère de l'éducation de l'Ontario (2007). Le curriculum de l'Ontario de $12^{\mathrm{e}}$ année. Français. Cours préuniversitaire, 88. http://www.edu.gov.on.ca/fre/curriculum/secondary/francais.html

Moffet, J.-D. (1995). Des stratégies pour favoriser le transfert des connaissances en écriture au collégial. Revue des sciences de l'éducation, 21(1), 95-120.

Monballin, M. (2011). L'acculturation à la communication scientifique : nécessité et embûches. Correspondance, 16(3), 1-6.

Monballin, M. et Legros, G. (2001). La maitrise langagière à l'entrée des études supérieures: mythes, constats et essais. Correspondance, 6(4), 1-8.

Monballin, M., van der Brempt, M. et Legros, G. (1995). Maîtriser le français écrit à l'université : un simple problème de langue? Revue des sciences de l'éducation, 21(1), 59-74.

Piron, S. (2008). La maitrise du français écrit : où le bât blesse. Correspondance, 14(1), 1-8.

Pilote, A. et Magnan, M.-O. (2008). L'école de la minorité francophone : l'institution à l'épreuve des acteurs. Dans J.-Y Thériault, A. Gilbert et L. Cardinal (Dir.). L'espace francophone en milieu minoritaire au Canada. Nouveaux enjeux, nouvelles mobilisations. (p. 275-317). Québec : Fides.

Roy, G.-R. (1995). Résolution de problèmes d'ordre syntaxique par des étudiants du postsecondaire. Revue des sciences de l'éducation, 21(1), 168-195.

Roy, G.-R. (1992). Vers un triple regard sur le français écrit des étudiants de collèges et d'universités. Sherbrooke : Éditions du CRP.

Roy, G.-R. et Boudreau, G. (1995). Des objectifs de l'enseignement du français à la pratique discursive : quinze ans plus tard. Revue des sciences de l'éducation, 21(1), 5-16.

Roy, G.-R. et Lafontaine, L. (1992). Étude de la maitrise du français écrit à l'université. Sherbrooke : Éditions du CRP. 
Roy, G.-R. et Legros, C. (1995). Du discours métalinguistique tenu par des étudiants du postscondaire relativement aux accords en genre et en nombre. Cahiers de la recherche en éducation, 2(2), 227-257.

Simard, C. (1995). L'orthographe d'usage chez les étudiants des ordres postsecondaires. Revue des sciences de l'éducation, 21(1), 145-165.

Vandendorpe, C. (1995). Apprendre à écrire à l'Université. Pour une approche contrastive. Dans J.-Y Boyer, J.-P Dionne et P. Raymond (Dir.). La production de textes. Vers un modèle d'enseignement de l'écriture (p. 301-331). Montréal : Logiques.

Viau, R. (1995). Le profil motivationnel d'étudiants de collèges et d'universités au regard du français écrit. Revue des sciences de l'éducation, 21(1), 197-215. 